\title{
Commentary: Is It Permissible If the Patient Asks About Herbal Medicine-Red Ginger to Relieve Women's Urinary Tract Infections?
}

\author{
Rara Sagita1, Eighty Mardiyan Kurniawati2*, \\ Zakiyatul Faizah ${ }^{3}$, Nur Anisah Rahmawati4 \\ ${ }^{1}$ School of Midwifery, Faculty of Medicine, Universitas Airlangga, Surabaya, Indonesia \\ ${ }^{2}$ Department of Obstetrics and Gynecology, Faculty of Medicine, \\ Universitas Airlangga, Surabaya, Indonesia \\ ${ }^{3}$ Department of Medical Biology, Faculty of Medicine, Universitas Airlangga, Surabaya, Indonesia \\ ${ }^{4}$ Faculty of Public Health, Universitas Airlangga, Surabaya, Indonesia
}

*Corresponding author details: Eighty Mardiyan Kurniawati; eighty-m-k@fk.unair.ac.id

\begin{abstract}
INTRODUCTION
Urinary tract infection is the most common outpatient infection, with a lifetime incidence of $50-60 \%$ in adult women. The prevalence of UTIs increases with age, and in women over the age of 65 is approximately double the rate seen in the overall female population [1]. Urinary tract infections (UTIs) are one of the leading causes of morbidity and comorbidity in patients with underlying conditions, and constitute the majority of reasons for hospital visits globally [2]
\end{abstract}

A common health care problem worldwide, urinary tract infection (UTI), is a disease that has a significant impact on the economy of every country, being the most common cause of hospitalization among the elderly and the most common cause of antibiotic prescription in primary care [3]. Healthcare-associated UTIs are the most common form of healthcare-acquired infection. UTIs create a significant social and personal burden, with a large number of medical visits in the United States each year associated with UTIs. In addition, measures of quality of life significantly impact women with recurrent UTIs [1]. Urinary tract infection (UTI) is a condition that is often complained of by women both in the general public and in hospitals. It has been estimated that one in three women will experience at least one episode of UTI in their lifetime [4]. Urinary tract infections (UTIs) disproportionately affect female adults. Postmenopausal women are particularly susceptible to recurrent UTI. Current prevalence estimates are lacking, despite the significant negative effects of recurrent UTIs on quality of life and health care costs [5]

The etiology in this age group varies according to health status with factors such as catheterization influencing the likelihood of infection and the pathogen most likely responsible. In younger women, increased sexual activity is a major risk factor for UTI and recurrence within 6 months is common. European data show that repeated infections are associated with increased doctor absenteeism and visits [1]. Good knowledge of the factors associated with UTI allows timely intervention that can easily control the disease. Escherichia coli is the most common uropathogenic bacteria followed by Staphylococcus aureus, Klebsiella pneumoniae, Klebsiella oxytoca, Proteus mirabilis, Enterococcus faecalis, and Proteus vulgaris. Age 19 years, female sex, married individuals, genitourinary tract abnormalities, diabetes, hospitalization, catheter $<6$ days, and catheter stay $>6$ days had a statistically significant relationship with UTI [2]. For unknown host factors, possibly genetic, women predisposed to recurrent urinary tract infection (RUTI) have a tendency to have longer and more severe colonization with uropathogens [19].

Diagnosing and managing upper and lower UTIs has always been a challenge for clinicians, given their high prevalence, risk of recurrence and inappropriate treatment, and the fact that antibiotic resistance is increasing worldwide, which necessitates the adoption of appropriate antibiotic stewardship. In addition to the various usual antibiotic regimens for treating UTIs, a large number of non-antimicrobial treatment modalities are highlighted including the new use of antibiotics and intravesical vaccines for the treatment of suppression. Finally, a pathway is suggested for proper diagnosis and treatment that ensures the stewardship of antibiotics to reduce long-term complications [3].

\section{URGENT CALL FOR THERAPY TO WOMEN'S URINARY} TRACT INFECTIONS

The first problem in the management of urinary tract infections begins with the diagnosis of the case. The diagnostic error for UTI in the ED has been reported to be as high as $30-50 \%$. While women of childbearing age who present with the classic symptoms of dysuria, frequency, and hematuria have a high probability of disease, results in the more general cohort of patients are both physically and laboratory less accurate [7]. Management problems such as inappropriate antibiotic prescribing is common for the treatment of UTIs. Clinical practice guidelines (CPG) are not associated with clinically significant changes in national antibiotic prescribing practices for UTIs in the USA [6]. In addition to the problem of RUTI, a review of individual expert and community recommendations underscores the lack of uniformity and the need for a more robust and generally acceptable definition of RUTI for use in clinical and academic practice [8].

There is significant variation in patient care associated with inconsistent recommendations from major professional societies due to a lack of high-quality evidence supporting clinical guidelines. Most women with recurrent UTIs initially present to their primary care physician for symptomatic management of UTIs. 
Although many adult women recall occasional and infrequent UTIs, the majority of patients with recurrent UTIs report a distinct change in the frequency of UTIs. Initial evaluation and treatment plan may be informed by reports of common life events associated with increased UTI frequency, including sexual debut, new sexual partners, new forms of contraception, urinary catheter use, pelvic surgery, menopausal oestrogen loss, faecal incontinence or diarrhoea, or onset of symptoms. significant. a medical condition (e.g., immunosuppression, diabetes, neurological disease that affects bladder emptying) or related medications. In addition to a focused history and physical examination, the initial evaluation of an adult woman with recurrent UTIs should explain the occurrence of frequent UTIs; details of UTI events, including UTI test results if available; previous treatment; antibiotic allergy; prevention and treatment preferences (antibiotics and alternative antibiotics); and current preventive practices, such as avoiding postcoital and drinking more water (2-3 L/day) [5].

In the assessment of early symptoms, it is necessary to understand the cause of UTI. If predisposing factors are not identified and eliminated, UTI can lead to more serious consequences, particularly kidney damage and kidney failure. A good understanding of the possible pathogenic factors that contribute to the development of UTIs and their recurrence will help the GP to interview patients, search for causes that are still undiscovered, and to identify appropriate therapeutic strategies [9]. Strategies in the medical management of urinary tract infections involve antimicrobial and non-antimicrobial strategies. Nonantimicrobial prophylactic strategies offer the opportunity to reduce both the rate of UTI and the patient's experience of personal burden [1]. Antimicrobial stewardship interventions are needed to increase outpatient antibiotic prescribing for uncomplicated UTIs [6].

\section{HERBAL MEDICINE- MAGNETISM AND POTENCY}

The cornerstone of treatment for any bacterial infection, including UTI, is antimicrobial therapy. Because UTIs are so common, especially in women, controlled use of antibiotics should be initiated for treatment. The type and duration of antimicrobial therapy depend on the location and severity of the infection and host/bacterial factors. Common treatment strategies for eliminating infection are judicious use of antibiotics, ensuring adequate hydration, and eliminating urinary tract obstruction or foreign bodies [3]. Given the increasing antibiotic resistance and increasing use of delayed prescribing for uncomplicated lower urinary tract infections (UTIs), patients at risk of treatment failure should be identified early [11].

Urinary tract infections are very common and result in significant patient morbidity and huge financial costs for the health care system. Recurrent urinary tract infections can be a challenge for many healthcare professionals, and repeated use of antibiotics in this group of patients inevitably contributes to the growing problem of antimicrobial resistance and superbugs [12]. The use of different prophylactic regimens and alternative strategies are available to reduce antibiotic exposure [10]. Although standard UTI therapy is initiated with antimicrobial therapy, alternative strategies are available to reduce antibiotic exposure, such as the use of methenamine salts, probiotics, cranberry juice, immunoprophylaxis, and vaginal oestrogen in postmenopausal women [10].

Recurrent acute cystitis is an important women's health problem. Frequent re-infection is frustrating and bothers many women, although there are no long-term negative outcomes. Management includes appropriate treatment for each symptom episode, and the establishment of strategies to reduce the frequency of infection in the long term.
Currently, the most appropriate long-term strategy is antimicrobial prophylaxis for women with frequent infections, or self-medication for women with less frequent infections. Any management approach should be developed in consultation with individual women with recurrent infections [13].

One of the developments of treatment is related to herbal medicine. Biodiversity is defined as the diversity of all life forms on earth, along with the interactions between them and their physical environment. As an archipelagic country with thousands of islands, Indonesia is blessed with rich and unique biodiversity. Indonesia's tropical forests cover about 143 million hectares and are home to about $80 \%$ of the world's medicinal plants. Indonesia's tropical forests are estimated to have 28,000 plant species. There are various reports on higher plant inventories in Indonesia. The Indonesian Country Study on Biodiversity (ICSBD 1993) estimates the number of flowering plant species in Indonesia to be between 25,000 and 30,000. Around 40 million Indonesians have historically used herbal medicines for the protection and treatment of disease and Indonesians have used around 6000 species of plants. Data on the number of medicinal plants also varies. Herbal medicine is and will remain an integral part of the Indonesian healthcare system. In vitro, in vivo and clinical studies on medicinal plants used in herbal medicine have partially scientifically proven their claimed biological activity. Species belonging to the Zingiberaceae family such as Curcuma, Zingiber, Kaempferia, are the plants most often used in the manufacture of herbal medicine. This species has also been studied intensively for its secondary metabolites and biological activities [14].

Ginger is commonly used in herbal medicine and has antioxidant and anti-inflammatory effects on women's reproductive health due to its content of flavonoids, phenolic compounds and active antioxidants such as shagaols, gingerols, zingerone. [18]. Red ginger can be used as an antimicrobial because of its ability to inhibit microbial growth. On the ability of phenol to denature protein [19]. Fresh and dried red ginger water extract has analgesic activity but its activity decreases with time [20].

Urinary tract infections are mostly caused by Escherichia Coli bacteria. There is antimicrobial activity in red ginger even with different types of applications and it can be combined with other plants. The compounds contained in ginger that can inhibit microbial growth consist of phenolic compounds, flavonoids, terpenoids and essential oils. Red ginger used to inhibit the activity of $E$. coli bacteria contains tannins, saponins, phenolic compounds, essential oils and flavonoids. Another study was also conducted by Tabassum et al (2013) which stated that phytochemical research found that compounds in red ginger contain flavonoids, phenolics, alkaloids and tannins [15].

E. coli bacteria that cause urinary tract infections (UTI) can be inhibited with red ginger extract and the higher the concentration, the higher the antibacterial activity obtained. This is the same as other studies that observed that ginger oil can inhibit $70 \%$ of $E$. coli bacteria by diluting ginger as much as $0.06 \%$, this can be seen from the Minimum Inhibitory Concentration (KMH) test on bacteria, which can be concluded from this study. This indicated that ginger oil showed antibacterial activity on the isolates of $E$. coli bacteria. Ginger can be used as an antibacterial to reduce the impact of UTI, because it contains $3.9 \%$ essential oil, some of which contain chemicals. including oleorosin and essential oils as the main components and have volatile oil and nonvolite oil, flavonoids can inhibit the permeability of bacterial cell 
walls, alkaloids can interfere with peptidoglycan constituent components in cells, and saponins can damage microbial cell membranes [16]. Urinary tract infections and wounds given red ginger extract showed antibacterial activity [17].

\section{WHAT DO WE NEED TO DO AND DEVELOP IN RESEARCH AND CLINICAL CHOICE?}

Red ginger has antimicrobial activity which can be useful in the management of urinary tract infections but it is necessary to clarify more about how to apply ginger properly. Suggestions for further researchers are to increase database searches so that more articles are obtained for literature studies as well as more in-depth and homogeneous studies in terms of participants, demographic characteristics, and public acceptance of the effect of giving red ginger as a treatment for urinary tract infections. In diagnosis, an accurate approach is needed so as to be able to detect cases correctly so that the treatment given is appropriate and the treatment is commonly used.

\section{CONCLUSION}

Patients can be given permissible if the patient asks about herbal medicine-red ginger to relieve women's urinary tract infections. This can be a support for medical management. In addition, it may be able to bring its own confidence and comfort for the patient.

\section{ETHICAL APPROVAL}

Not applicable

\section{FUNDING SOURCES}

None received

\section{DECLARATION OF COMPETING INTEREST}

None declared

\section{REFERENCES}

[1] M. Medina and E. Castillo-Pino, "An introduction to the epidemiology and burden of urinary tract infections," Ther. Adv. Urol., vol. 11, p. 1756287219832172, Jan. 2019, doi: $10.1177 / 1756287219832172$.

[2] M. Odoki et al., "Prevalence of Bacterial Urinary Tract Infections and Associated Factors among Patients Attending Hospitals in Bushenyi District, Uganda," Int. J. Microbiol., vol. 2019, p. 4246780, 2019, doi: 10.1155/2019/4246780.

[3] N. F. Abou Heidar, J. A. Degheili, A. A. Yacoubian, and R. B. Khauli, "Management of urinary tract infection in women: A practical approach for everyday practice," Urol. Ann., vol. 11, no. 4, pp. 339-346, 2019, doi: 10.4103/UA.UA_104_19.

[4] S. Salvatore et al., "Urinary tract infections in women," Eur. J. Obstet. Gynecol. Reprod. Biol., vol. 156, no. 2, pp. 131-136, 2011, doi: https://doi.org/10.1016/j.ejogrb.2011.01.028.

[5] S. Aslam, M. Albo, and L. Brubaker, "Recurrent Urinary Tract Infections in Adult Women," JAMA, vol 323, Jan. 2020, doi: 10.1001/jama.2019.21377.

[6] M. J. Durkin et al., "An Assessment of Inappropriate Antibiotic Use and Guideline Adherence for Uncomplicated Urinary Tract Infections," Open Forum Infect. Dis., vol. 5, no. 9, p. ofy198, Sep. 2018, doi: 10.1093/ofid/ofy198.

[7] R. A. Taylor, C. L. Moore, K.-H. Cheung, and C. Brandt, "Predicting urinary tract infections in the emergency department with machine learning," PLoS One, vol. 13, no. 3, p. e0194085, Mar. 2018, [Online]. Available: https://doi.org/10.1371/journal.pone.0194085.
[8] R. D. Malik, Y. (Rose) Wu, and P. E. Zimmern, "Definition of Recurrent Urinary Tract Infections in Women: Which One to Adopt?" Female Pelvic Med. Reconstr. Surg., vol. 24, no. 6, 2018, [Online]. Available:

https://journals.lww.com/fpmrs/Fulltext/2018/11 000/Definition_of_Recurrent_Urinary_Tract_Infectio ns.8.aspx.

[9] D. Minardi, G. d'Anzeo, D. Cantoro, A. Conti, and G. Muzzonigro, "Urinary tract infections in women: etiology and treatment options," Int. J. Gen. Med., vol. 4, pp. 333-343, 2011, doi: 10.2147/IJGM.S11767.

[10] A. Al-Badr and G. Al-Shaikh, "Recurrent Urinary Tract Infections Management in Women: A review," Sultan Qaboos Univ. Med. J., vol. 13, no. 3, pp. 359-367, Aug. 2013, doi: 10.12816/0003256.

[11] R. Martischang et al., "Risk factors for treatment failure in women with uncomplicated lower urinary tract infection," PLoS One, vol. 16, no. 8, p. e0256464, Aug. 2021, [Online]. Available: https://doi.org/10.1371/journal.pone.0256464.

[12] N. Sihra, S. Malde, T. Greenwell, M. Pakzad, M. Kujawa, and A. Sinclair, "Management of recurrent urinary tract infections in women," J. Clin. Urol., p. 2051415820939456, Jul. 2020, doi: $10.1177 / 2051415820939456$.

[13] L. E. Nicolle, "Managing Recurrent Urinary Tract Infections in Women," Women's Heal., vol. 1, no. 1, pp. 39-50, Jul. 2005, doi: 10.1517/17455057.1.1.039.

[14] Elfahmi, H. J. Woerdenbag, and O. Kayser, "Jamu: Indonesian traditional herbal medicine towards rational phytopharmacological use," J. Herb. Med., vol. 4, no. 2, pp. 51-73, 2014, doi: https://doi.org/10.1016/j.hermed.2014.01.002.

[15] H. Tabassum, M. N. Ali, N. Al-Jameil, and F. A. Khan, "Evaluation of antibacterial potential of selected plant extracts on bacterial pathogens isolated from urinary tract infections.," Int. J. Curr. Microbiol. Appl. Sci., vol. 2, no. 10, pp. 353-368, 2013, [Online]. Available: http://www.ijcmas.com/vol-2-10/Hajera Tabassum, et al.pdf.

[16] Tim Lentera., Khasiat dan Manfaat Jahe Merah Si Rimpang Ajaib. Jakarta: AgroMedia, 2015.

[17] A. P. K. Y. Al-zamily, L. Riyadh, and H. Wally, "Effect of Watery Ginger Extract on some Bacteria Isolated from Urinary Tract Infections and Compared to Antibiotics," no. 1, 2017, doi: 10.18081/11124123/017-4/112-118.

[18] R. Yasmin, D. Pertiwi, and R. Rahmawati, "the Role of Ginger Administration Toward Oxidative Stress in Women'S Health Reproduction: a Literature Review," Bunda Edu-Midwifery J., vol. 4, no. 2, pp. 25-29, 2021, doi: $10.54100 /$ bemj. v4i2.52.

[19] P. Hajishafiee, M., Azadbakht, L., \& Adibi, "Energy and nutrient requirements in the intensive care unit inpatients: A narrative review," JNSD, vol. 1, no. 2, pp. 63-70, 2015.

[20] Y. Febriani, H. Riasari, W. Winingsih, L. Aulifa, and A. Permatasari, "Potensi Pemanfaatan Jahe Merah (Zingiber officinale Roscoe) sebagai Obat Analgetik," Indones. J. Pharm. Sci. Technol., vol. 1, no. 1, pp. 5764, 2018. 